OPEN ACCESS

Edited by:

Heiko Hamann,

University of Lübeck, Germany

Reviewed by:

Payam Zahadat,

University of Graz, Austria

Mark Post

University of York, United Kingdom

Gabriele Valentini,

Arizona State University,

United States

*Correspondence:

Carlos Gershenson cgg@unam.mx

Specialty section: This article was submitted to Computational Intelligence in

Robotics,

a section of the journal Frontiers in Robotics and $A$

Received: 16 November 2019 Accepted: 09 March 2020

Published: 03 April 2020

Citation:

Gershenson C (2020) Guiding the Self-Organization of Cyber-Physical Systems. Front. Robot. Al 7:41. doi: 10.3389/frobt.2020.00041

\section{Guiding the Self-Organization of Cyber-Physical Systems}

\author{
Carlos Gershenson ${ }^{1,2,3 *}$ \\ ${ }^{1}$ Departamento de Ciencias de la Computación, Instituto de Investigaciones en Matemáticas Aplicadas y en Sistemas, \\ Universidad Nacional Autónoma de México, Mexico City, Mexico, ${ }^{2}$ Centro de Ciencias de la Complejidad, Universidad \\ Nacional Autónoma de México, Mexico City, Mexico, ${ }^{3}$ ITMO University, St Petersburg, Russia
}

Self-organization offers a promising approach for designing adaptive systems. Given the inherent complexity of most cyber-physical systems, adaptivity is desired, as predictability is limited. Here I summarize different concepts and approaches that can facilitate self-organization in cyber-physical systems, and thus be exploited for design. Then I mention real-world examples of systems where self-organization has managed to provide solutions that outperform classical approaches, in particular related to urban mobility. Finally, I identify when a centralized, distributed, or self-organizing control is more appropriate.

Keywords: complexity, self-organization, information, adaptation, robustness, antifragility

\section{INTRODUCTION}

We are submerged in complexity. And this complexity is increasing. But what is complexity? There are dozens of definitions and measures in the literature (Lloyd, 2001; Gershenson and Heylighen, 2005), but not a definite one. Well, life is not properly defined either, and it is not a hindrance for biology. Still, to have an idea of what we refer to, let us go to its etymological root. Complexity comes from the Latin plexus, which means entwined. In other words, something complex is difficult to separate. This is because the interactions among its components are relevant (Gershenson, 2013b). Relevant because they co-determine the future of the system. Thus, if we do not consider such interactions, but study components in isolation, we will not be able to understand the system properly. Also, interactions can generate novel information, not present in initial nor boundary conditions. This novel information limits predictability (Gershenson, 2013a) and is the source of computational irreducibility (Wolfram, 2002), i.e., there is no shortcut to know the future: one must go through all intermediate steps, because the information produced in the process is required to reach/compute the future.

A recent collaborative effort produced this definition: "Complexity science, also called complex systems science, studies how a large collection of components-locally interacting with each other at small scales-can spontaneously self-organize to exhibit non-trivial global structures and behaviors at larger scales, often without external intervention, central authorities or leaders. The properties of the collection may not be understood or predicted from the full knowledge of its constituents alone. Such a collection is called a complex system and it requires new mathematical frameworks and scientific methodologies for its investigation." (De Domenico et al., 2019).

One of the core concepts explained in De Domenico et al. (2019) is self-organization: "Interactions between components of a complex system may produce a global pattern or behavior. This is often described as self-organization, as there is no central or external controller. Rather, the "control" of a self-organizing system is distributed across components and integrated through their interactions. Self-organization may produce physical/functional structures like crystalline 
patterns of materials and morphologies of living organisms, or dynamic/informational behaviors like shoaling behaviors of fish and electrical pulses propagating in animal muscles. As the system becomes more organized by this process, new interaction patterns may emerge over time, potentially leading to the production of greater complexity." Common examples of selforganizing systems include flocks of birds, schools of fishes, insect swarms, herds, crowds, and other collective phenomena (Camazine et al., 2003; Vicsek and Zafeiris, 2012), although self-organization is not restricted to living systems (Nicolis and Prigogine, 1977; Haken, 1988; Gershenson and Heylighen, 2003; Prokopenko et al., 2009).

There are many cases where self-organization has been used as an approach in engineering (Di Marzo Serugendo et al., 2004; De Wolf et al., 2005; Zambonelli and Rana, 2005; Mamei et al., 2006; Helbing et al., 2007; Dressler, 2008; Müller-Schloer et al., 2011; Rohden et al., 2012; Brambilla et al., 2013; Rubenstein et al., 2014; Vásárhelyi et al., 2018). In these cases, we can describe a system as self-organizing when elements interact to achieve dynamically a global function or behavior (Gershenson, 2007). In other words, instead of designing directly a solution, one regulates the potential interactions among elements. This is useful in non-stationary problems: when the situation changes, then the system adapts by itself. Since interactions in complex systems produce novel information, it is common that this information will change a complex problem. Not only its state, but also its state space. Thus, self-organization can be useful to face complexity by providing general adaptation mechanisms. Several methodologies using self-organization have been proposed (see Frei and Di Marzo Serugendo, 2011 for an overview), although the approach has not been widely applied.

In a parallel effort, guided self-organization attempts to combine seemingly opposed processes: design to define and regulate the properties and behavior of a system (one tells the system what to do), and self-organization that implies certain autonomy and adaptability (the system follows its own dynamics) (Prokopenko, 2009, 2014; Ay et al., 2012; Polani et al., 2013). Guided self-organization can be understood as "the steering of the self-organizing dynamics of a system toward a desired configuration" (Gershenson, 2012).

In this paper, I compile concepts and approaches useful for designing self-organizing systems in the physical realm. I illustrate these with case studies from urban mobility before discussing implications. A diagram of the paper structure is shown in Figure 1.

\section{CONCEPTS}

Several concepts are useful to design and guide self-organizing systems. In this section, a non-exhaustive list is presented.

\subsection{Adaptation}

Adaptation can be defined as a change in an agent or system as a response to a state of its environment that will help the agent or system to fulfill its goals (Gershenson, 2007). Living systems naturally adapt to changes in their environment, and artificial systems can benefit from exhibiting adaptation (Holland, 1975; Steels and Brooks, 1995; Bedau et al., 2013).

If problems are stationary, i.e., do not change, then it is worthwhile attempting to predict the future of a system to control it. However, for non-stationary problems, predictability by definition is limited. Novel information generated by interactions in complex systems can lead to non-stationarity. In this case, adaptation is desirable to complement the unpredictable aspects of a problem (Gershenson, 2013a). And self-organization offers a method for building adaptive systems.

For example, city traffic is changing constantly: every time a red light switches to green, the number of waiting vehicles is different. Thus, the timing of the traffic lights should also change to prevent idling. Traditional adaptive traffic light control methods (e.g., Sydney, Dublin, Singapore) use sensors to shift phases depending on recent average demands. This is usually better than not having adaptation, where the best possible option would be to take average measurements, set fixed phases, and perhaps change the programs a few times per day. However, if traffic lights can adapt at the same timescale as the traffic demand does, i.e., every cycle, then the performance would be much improved (Goel et al., 2017).

Adaptation implies flexibility and can take place at different timescales: learning is relatively fast, development occurs during the lifetime of an individual, and evolution acts across generations.

\subsection{Robustness}

A system is robust if it continues to function in the face of perturbations (Wagner, 2005), and in general any type of change. As with adaptation, robustness is prevalent in living systems and desirable in artificial ones (Jen, 2005).

Robustness and adaptability are complementary: a system has to be robust enough to survive while it adapts, and adaptation can favor robustness.

For example, the Internet is quite robust. The TCP/IP protocol was designed to resist nuclear warfare. If any server goes down, other servers will manage to transmit packages, unless the network becomes disconnected. At the structural level (which servers are linked, which pages are linked), self-organization has led to a scale-free topology (Barabási et al., 2000), which is also robust to random failures (although fragile to directed attacks Caldarelli, 2007). This is because only few nodes have several connections, so most probably a random failure will affect a non-important node. However, directed attacks can aim for the hubs.

Robust systems are more prone to be scalable than fragile ones. Adding new components or functionality to a system can be seen as a type of perturbation, so in this sense robustness becomes a requirement for scalability.

\subsection{Antifragility}

A fragile system is damaged by perturbations. A robust system is unaffected by perturbations. An antifragile system benefits from perturbations (Taleb, 2012). Particular examples of systems that benefit from noise had been already identified (Atlan, 1974), and the concept of antifragility can be seen as a generalization. 


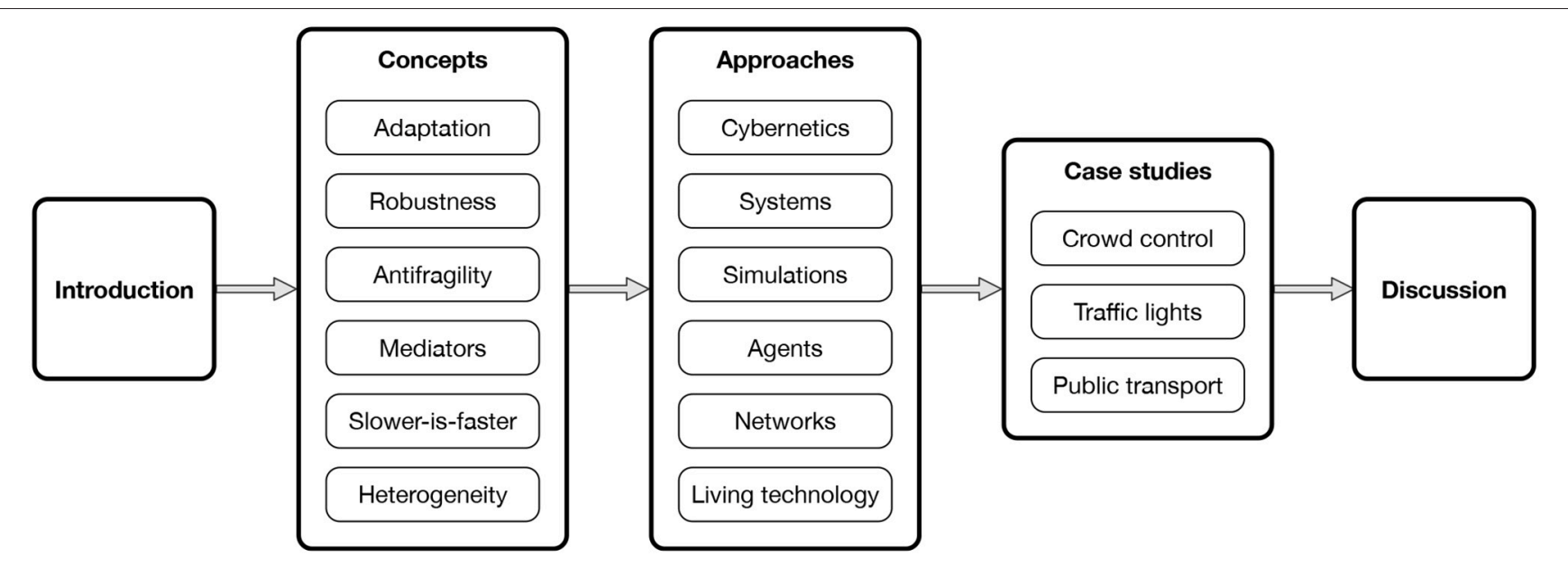

FIGURE 1 | Diagram of the paper structure depicting sections and subsections.

For example, the immune system is antifragile. Children who grow up in extremely sanitized conditions are not exposed to pathogens (perturbations), so their immune systems do not develop, leading to stronger infections and allergies in adulthood. Certainly, children should not be infected intentionally, but being exposed to a "normal" amount of pathogens and falling ill now and then is helpful for training the immune system.

We have recently proposed a measure of antifragility (Pineda et al., 2019), which is positive when perturbations improve the performance of a system, negative when perturbations decrease the performance (fragility), and zero when perturbations do not affect the performance (robustness). An important aspect is that there is no "optimal" antifragility independent of an environment. A system should be as antifragile as its environment varies (this is related with requisite variety, discussed in section 3.1).

\subsection{Mediators}

Interactions can be classified as positive, neutral, or negative, depending on the effect they have on the goals of a system (Gershenson, 2007, 2011b).

A mediator arbitrates among the elements of a system, to minimize conflict, interferences and frictions (negative interactions); and to maximize cooperation and synergy (positive interactions) (Michod, 2003; Heylighen, 2006; Gershenson, 2007).

Negative interactions, by definition, are those that prevent or damage the functionality, performance, goals, or behavior of a system. Positive interactions would benefit, facilitate, or promote them. Neutral interactions do not affect them. For example, actions that generate a cost but fail to provide a benefit for a society can be said to generate friction, e.g., aggression. If the benefit provided by actions is greater than the cost, one can say that they are synergistic, e.g., politeness. If the cost and benefit balance out, the interactions would be neutral, e.g., tolerance.

Traffic rules can be seen as examples of mediators. They aim at reducing conflict in urban mobility. Without these rules, we would need to decide constantly on which side of the streets to drive, how to give way, make turns, etc. Even when rules and norms vary from country to country, and in some cases from city to city, when everybody follows the same set of rules (mediators), conflicts tend to be reduced.

Money is another example. It mediates transactions that are much facilitated compared to bartering.

Designing mediators can be useful for regulating systems where the elements cannot be modified. Still, mediators can change the interactions between elements, leading to different systemic behavior and properties (see case study in section 4.1).

\subsection{Slower-Is-Faster Effect}

Probably this effect was first described about 20 years ago while modeling crowd dynamics (Helbing et al., 2000a,b). If people trying to evacuate a room are panicked (trying to exit faster), then they create friction (negative interactions) that leads to a "turbulent" flow that is slower than if people exit calmly (neutral interactions), thus with a "laminar" flow. The same effect has been studied in vehicular traffic, logistics, public transport, social dynamics, ecological systems, and adaptive processes (Gershenson and Helbing, 2015).

In general, the slower-is-faster effect occurs when a system performs worse as its components try to do better. This implies that a balance between doing "too few" and doing "too much" is necessary. However, in many cases this balance is dynamic, as with antifragility. For example, the optimal speed for highway traffic (that maximizes flow) depends on the vehicular density. For this reason, systems that present a sloweris-faster effect, require constant adaptation, that can be achieved through self-organization.

The slower-is-faster effect may refer to any variable, not only speed. For example, growth or profits are not necessarily maximized in the long term with a short-term maximization strategy. Managing natural resources, such as fisheries, requires this understanding: if all resources are depleted, then in the near future there will be no profits. Maximizing profits requires 
a careful balance between short-term action and long-term planning. As with the case of highway traffic, usually this balance is non-stationary.

\subsection{Heterogeneity}

Most of our models of complex systems are homogeneous: all components have the same properties. This simplification is useful when we face computational limitations. However, increasing processing power and data availability have allowed us to make more realistic models, where different elements of a system have varying properties.

Perhaps the most studied heterogeneity in complex systems is the one of network topologies (Albert and Barabási, 2002; Newman et al., 2006; Gershenson and Prokopenko, 2011; Barabási, 2016) (see section 3.5). Many networks are heterogeneous, with few elements having lots of connections and many elements having few connections. This leads to important differences with homogeneous, regular networks, where all elements have the same number of connections. Apart from the robustness already mentioned, heterogeneous networks can also transmit information faster (they have shorter average path lengths) (Aldana, 2003).

More recently, temporal heterogeneity has been also studied (Cocho et al., 2015; Morales et al., 2018), i.e., systems where different components change at different rates. In a similar way to structural heterogeneity, few elements change slower than most elements. This heterogeneity seems to lead to a balance where slow elements are robust and fast elements are adaptable. In homogeneous systems, this balance is achieved only in phase transitions, which can be characterized as "critical" (Balleza et al., 2008). However, heterogeneity seems to expand the balance beyond criticality, making it easier to search an unknown parameter space, simply because different components diversify any search procedure (Martínez-Arévalo et al., in preparation).

\section{APPROACHES}

How to implement the properties related to self-organization in cyber-physical systems? The concept of self-organizing systems originated within cybernetics (Ashby, 1947, 1962; von Foerster, 1960; Heylighen et al., 1993), where useful approaches were already developed.

\subsection{Cybernetics}

Ashby not only coined the term "self-organizing system," but he also proposed the law of requisite variety (Ashby, 1956; Heylighen and Joslyn, 2001; Bar-Yam, 2004; Gershenson, 2015). Variety can be understood as the possible number of states that a system can have. This law states that an active controller must have at least as much variety as the system it is trying to control. For example, if we want a robot at a manufacturing plant to deal with seven different types of boxes, then it should be able to distinguish and make the appropriate decisions to handle each type of box. A common problem is that complexity explodes variety and vice versa. Therefore, traditional (nonadaptive) approaches become limited. To handle the variety of a system, we can either reduce its variety (using mediators), or increase the variety of the controller, but then the latter will imply an increase in the complexity of the controller as well.

Everything else being equal, the variety of non-stationary domains will be greater or equal than those of stationary ones, as their change usually implies a greater number of potential states. Therefore, adaptive controllers and antifragile mechanisms have to consider this increased variety.

Active controllers are related with feedforward and feedback (positive or negative) control. Feedback occurs in response to a signal or perturbation, so it can be seen as a type of adaptation (Gershenson, 2007). Negative feedback reduces the effect of the perturbation, trying to reach stability, while positive feedback amplifies perturbations, leading to greater change. Feedforward control might be preferred, as it acts on a perturbation or signal before it can affect the controlled. However, this requires anticipation, and since complexity implies a limited predictability due to novel information being generated by relevant interactions (non-stationarity), this type of control will also be limited.

Complementary to active controllers, passive controllers were also studied in cybernetics, related to buffering. Passive control can increase the robustness of systems, since it prevents perturbations from affecting the controlled. Figure 2 illustrates active and passive controllers.

There is an interesting relationship between variety and heterogeneity. Heterogenous systems by definition have more variety, so in principle they should be able to control more situations than similar homogeneous systems. However, they might be less robust and more complicated to design and understand. For example, "if there is a system of ten agents each able to solve ten tasks, a homogeneous system will be able to solve ten tasks robustly (if we do not consider combinations as new tasks). A fully heterogeneous system would be able to solve a hundred tasks, but it would be fragile if one agent failed." (Gershenson, 2007, p. 53). In this case, the homogeneous system would be robust, because if one agent fails, others can perform the same function. Still, the variety of the system would be restricted to ten tasks. The heterogeneous system would have a tenfold variety, but if a single agent fails, then no other agent would be able to take over the task, and the system would fail as well. Thus, a balance between homogeneity and heterogeneity should also give us a balance between robustness and adaptability (Langton, 1990; Kauffman, 1993).

\subsection{Systems}

Contemporary and overlapped with cybernetics, systems theory has also permeated into all disciplines (von Bertalanffy, 1968). The word "system" comes from the ancient Greek $\sigma v \tilde{\sigma} \tau \epsilon \mu \alpha$ (sýstema), which means a whole made of several parts. It is a useful abstraction that can be applied to describe several phenomena at different scales. Moreover, it can be the basis for understanding how elements interact to generate behavior or properties at the system level, and how these properties regulate or constrain the behavior or properties of the elements.

Cybernetics and systems theory naturally merge in cyberphysical systems, where control and communication are required in the understanding and engineering of systems composed 


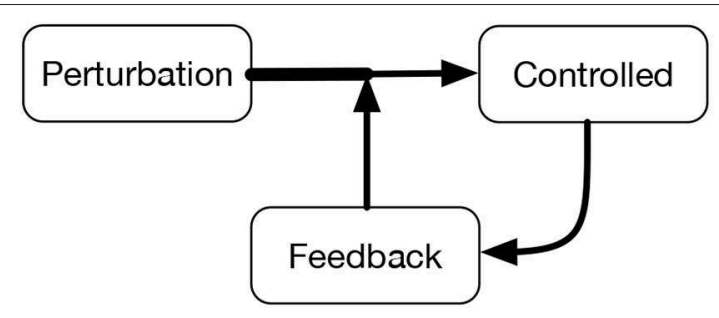

Active

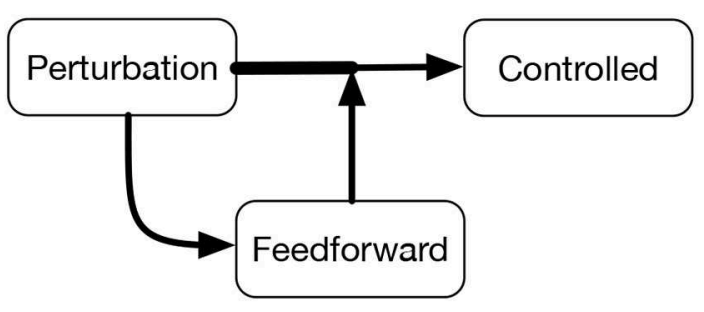

Active

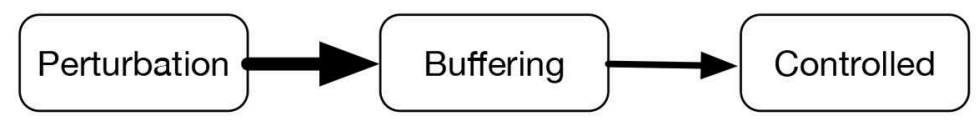

Passive

FIGURE 2 | Diagrams of feedback control, feedforward control, and buffering. In different ways, they try to reduce or eliminate the effect of perturbations on the controlled, either actively or passively. Arrows indicate the effects of perturbations: wider lines indicate greater effects. Ideally, the control mechanisms should be able to eliminate completely the effect of perturbations.

of "bits and atoms," i.e., digital information is entwined with physical mechanisms.

In a similar way, cyber-social systems are those that merge digital technology and social interactions. The "human factor" increases the variety of such systems, and our "creativity" limits even more their predictability.

\subsection{Simulations}

We can consider computers as telescopes of complexity (Pagels, 1989). In other words, without computers, our cognitive abilities are limited to studying models considering not many more than two or three variables. To explore models with thousands or millions of variables, computer simulations are necessary (Gershenson, 2007) because of computational irreducibility (Wolfram, 2002). Complexity implies that new information is generated by interactions, so there is no "shortcut" to the future and all intermediate steps are necessary (Wuensche and Lesser, 1992). This limits inherently the predictability of systems (Gershenson, 2013a).

Simulations do not replace other approaches, but their usefulness can be seen in the spreading of computational methods to all disciplines.

Also, simulations allow us to contrast theories in a synthetic way (Steels, 1993). The inductive method validates theories through observation of phenomena. The synthetic method builds artificial systems based on a theory, and then this is validated observing the performance of the artificial system (Simon, 1996).
Since one can contrast different theories using computer simulations, it can be said that computational social sciences are "hardening" the social sciences (Axelrod, 1997; Lazer et al., 2009).

\subsection{Agents}

Agent-based modeling (Bonabeau, 2002; Schweitzer, 2003; Epstein, 2006; Wilensky and Rand, 2015) has been a useful approach to describe complex systems. An agent can be defined as an entity that acts on its environment (Gershenson, 2007). As such, they can be used to model active controllers.

Agents have been used to model cognitive systems of different flavors, including rational (Wooldridge and Jennings, 1995), adaptive (Maes, 1994), social (Epstein and Axtell, 1996; Gershenson, 2001), and economic (Arthur, 1999; Challet et al., 2013).

Considering elements of a complex systems as agents, with states, goals, and rules allows us to study how changes at one scale lead to effects at another scale. The effects can go in both directions: changes in agents leading to changes in the system and vice versa. Moreover, systems can also be described as (higher scale) agents.

Another advantage of agent-based modeling is that such models are closer to common language than previous modeling approaches based in e.g., differential equations. Therefore, people do not require a strong mathematical background to develop models using a multi-agent approach. 


\subsection{Networks}

Another approach that is becoming more and more popular as data availability and computing power increase is network science (Newman, 2003; Newman et al., 2006; Barabási, 2016). Networks have the benefit of being able to represent naturally elements (nodes) and interactions (links). The relationship between the structure and function of networks has been an intense area of study, where self-organization can play a relevant role (Gershenson, 2012).

Different organizations of the same elements can lead to radically different functionalities. A classical example is different arrangements (allotropes) of carbon atoms, which can lead to charcoal, diamond, graphite, graphene, nanotubes, buckyballs, etc. The components are the same, but changing their organization (structure) leads to radically different properties (function) of these materials.

The robustness of systems can be promoted through different mechanisms (Gershenson, 2012), such as redundancy (having several copies of the same element), degeneracy (having different elements perform the same function), modularity (shortrange links stronger than long-range ones), and scale-free-like (heterogeneous) topologies (few elements with several links, several elements with few links).

\subsection{Living Technology}

Ethology - the study of animal behavior-has been taken as an inspiration to build adaptive systems (Beer, 1990; Maes, 1994; Steels and Brooks, 1995) and to study complex artificial systems (Rahwan et al., 2019). Animals have evolved to survive in complex environments, so adaptive strategies and self-organizing mechanisms found in nature have been used in cyber-physical systems. In this sense, living technology (Bedau et al., 2009; Gershenson et al., 2018) takes the advantageous properties of living systems and applies them in socio-technical systems, from protocells (Rasmussen et al., 2008) to cities (Gershenson, 2013c).

Living technology has been defined as technology that exhibits the properties of living systems, such as adaptation, learning, evolvability, robustness, and self-organization. Firstorder living technology is actually alive, either manipulating existing living systems (Gibson et al., 2010; Kriegman et al., 2020) or (eventually) building them from scratch (Rasmussen et al., 2008; Čejková et al., 2017). Second-order living technology uses living systems as components to achieve the desired properties found in living systems (Benyus, 1997; Liu and Tsui, 2006).

\section{CASE STUDIES}

In this section, I illustrate the previous concepts and approaches with case studies we have worked with in recent years, related to urban mobility. Particular concepts are highlighted, although approaches are implicitly used.

\subsection{Crowd Control}

More than a hundred million people use the hundred busiest metro systems in the world every day, a number that is growing fast as the urban population is increasing and cities develop. In the Mexico City Metro and other cyber-social systems, people would normally push each other, not letting passengers exit trains, collapsing the systems. How to regulate passenger behavior, when a selfish approach might seem to bring individual

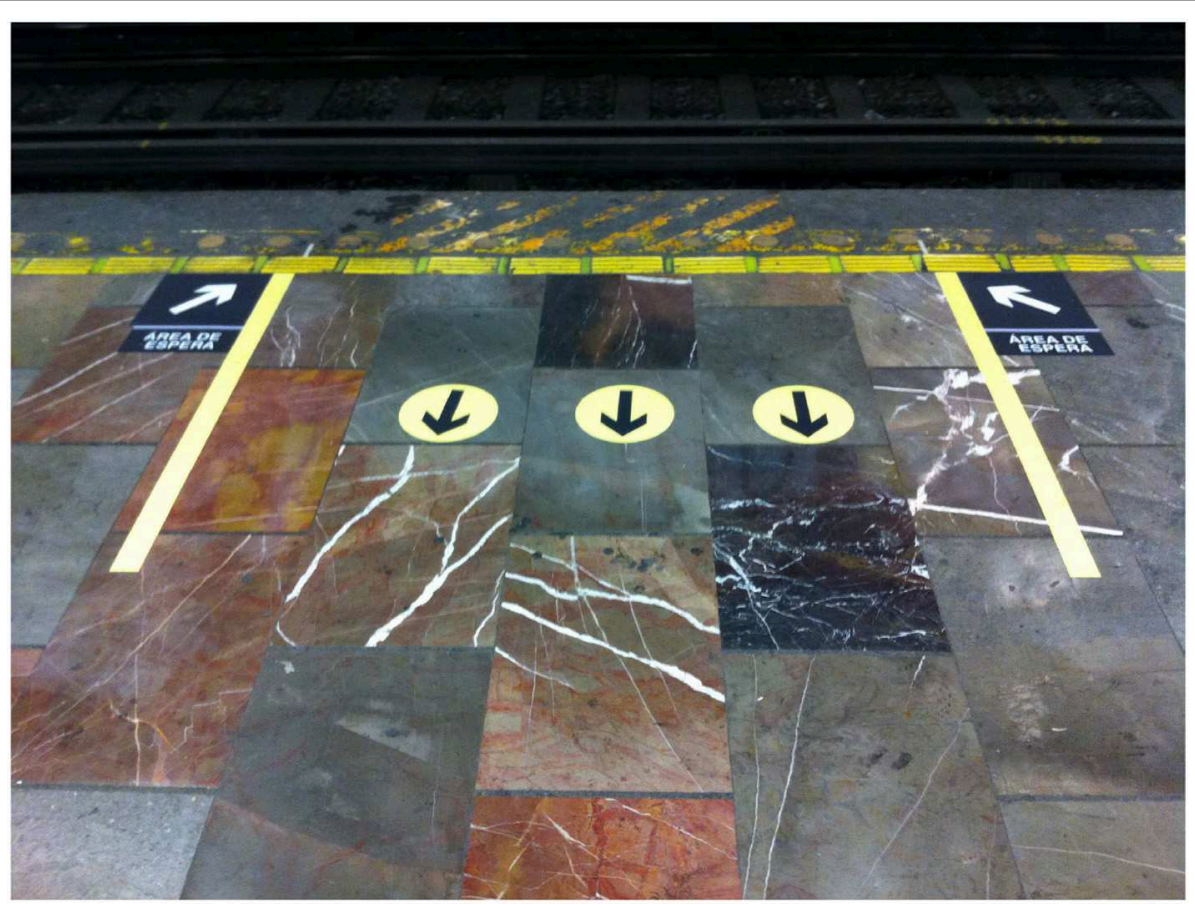

FIGURE 3 | Signs installed to mediate passenger boarding and descent in Mexico City Metro. Reproduced from Carreón et al. (2017) under the Creative Commons CCBY license https://doi.org/10.1371/journal.pone.0190100.g015. 
benefit but lead to collective inefficiency? One can think of different mediators, but they can be costly to try in real systems. To explore alternatives, we first used simulations of a model of crowd dynamics (Helbing et al., 2000a) and then implemented a pilot study in the Balderas station of the Mexico City Metro on December, 2016 (Carreón et al., 2017). The pilot was a success and it has since been extended to several other busy stations.

The intervention consisted of "simple" signs that indicate passengers roughly where the train doors will be, asking them to leave free space for exiting passengers, as shown in Figure 3. What we did not expect nor suggest was that people would queue (Figure 4), and that these queues could even go upstairs as people respected them.

This intervention managed to change the behavior of the passengers and thus the crowd, without changing the elements of the system (where could we get different "educated" passengers from?). The signs mediated interactions between people. This is an example of a passive control, where interactions are regulated "simply" providing useful information. The mediators managed to change the structure of the crowd, leading to a more efficient function.

\subsection{Traffic Light Coordination}

The coordination of traffic lights is an EXP-complete problem, meaning that in theory it takes exponentially more time to find a solution as more intersections are added to a street network. Also, the precise number of vehicles changes every cycle, so in practice the problem changes faster than it can be optimized. An active controller should adapt as fast as the controlled changes (requisite temporal variety), and for that sensors are required to provide relevant information to the controller.

With this in mind, we have proposed self-organizing algorithms that can coordinate traffic flows and adapt to constant changes in the demand as fast as it changes (Gershenson, 2005; Zapotecatl et al., 2017), achieving close-to-optimal performance (Gershenson and Rosenblueth, 2012). The main idea behind the algorithms is that streets with a higher demand get a preference. This is implemented by counting how many vehicles are approaching or waiting behind red lights, and when the integral over time of this counter reaches a threshold, then the green light is requested. Thus, busier directions will wait less for a green light. This increases the probability that vehicles will aggregate behind red lights with few cars, leading to the formation of platoons. As platoons reach a certain size, they can request a green light before they even reach an intersection (because they quickly reach the threshold), so vehicles do not need to stop, unless there are other vehicles or pedestrians crossing. Platoons are easier to coordinate than individual vehicles, as they leave spaces between them that other platoons can use without interference. When densities are high, the preference is given to the street that has more space after the intersection, preventing gridlocks.

It is difficult to compare the performance of self-organizing traffic lights, as there are no benchmarks in traffic light coordination. However, they are close to optimal. We can define optimality by calculating the maximum performance (measured in terms of velocity or flow) of isolated intersections for different densities. If a system with several intersections performs as efficient at every intersection, we can say that the coordination

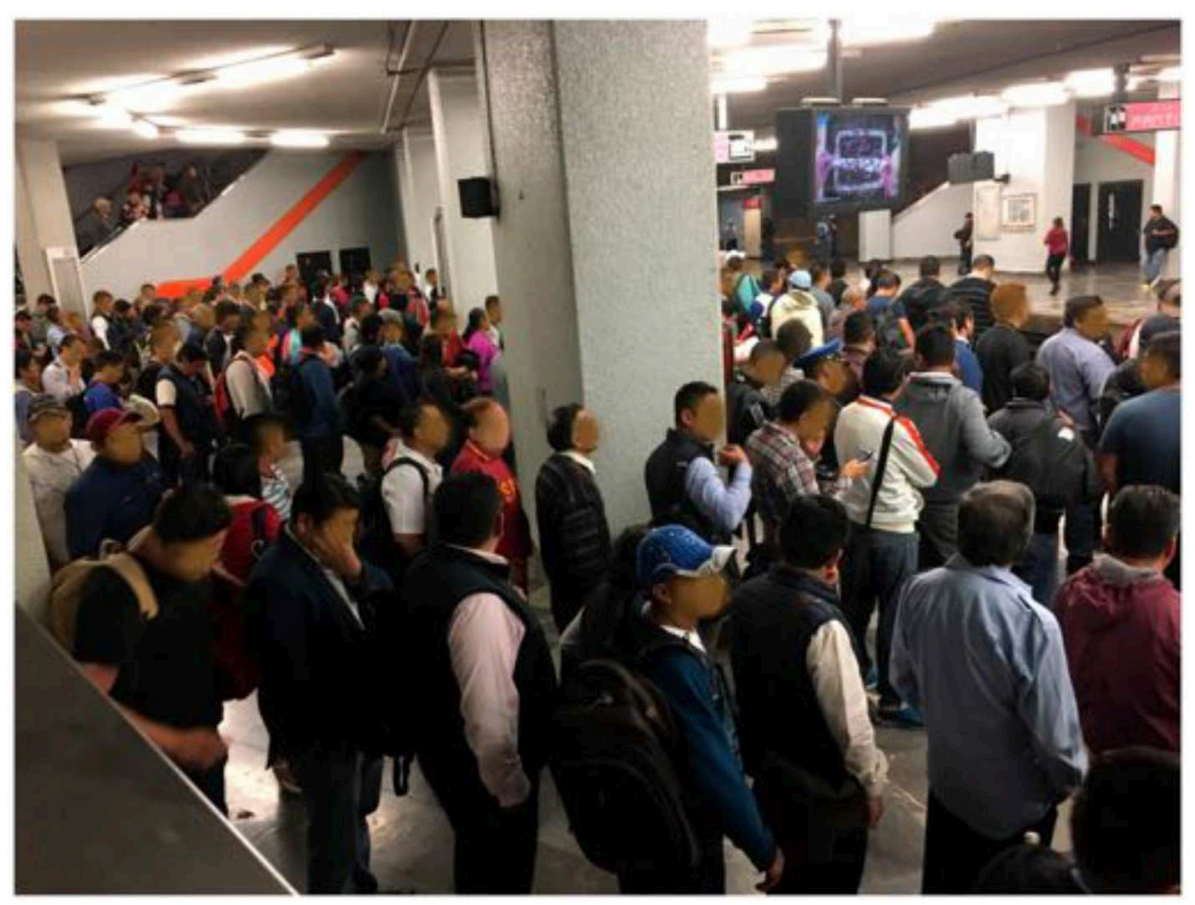

FIGURE 4 | Passengers queuing waiting for a train in Mexico City Metro during rush hour, San Lázaro metro station. Reproduced from Carreón et al. (2017) under the Creative Commons CCBY license https://doi.org/10.1371/journal.pone.0190100.g016. 

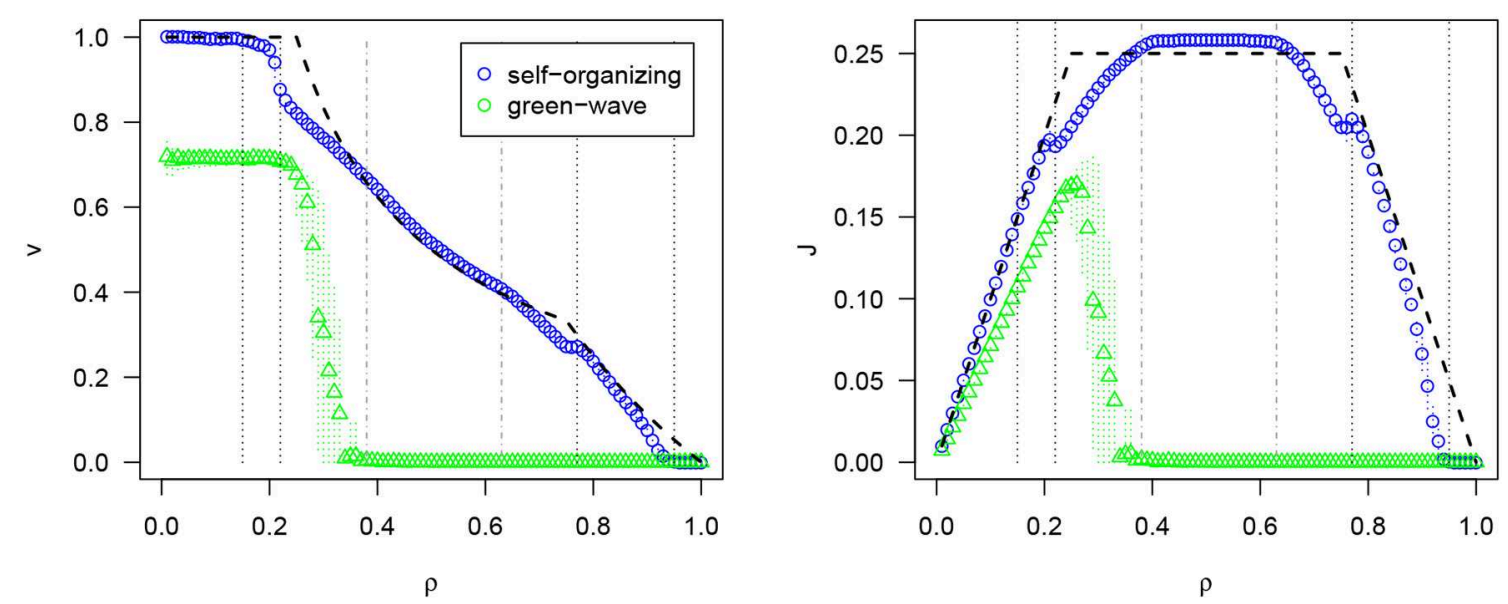

FIGURE 5 | Results of self-organizing traffic lights: average velocity $v$ and average flux $J$ for different densities $\rho$. Optimality curves shown with dashed black lines. Reproduced from Zubillaga et al. (2014) under the Creative Commons Attribution License.

is optimal. Figure 5 shows a comparison of the self-organizing approach and a traditional top-down control method known as the "green wave" that attempts to offset phases according to the expected speed of vehicles. However, demands change constantly and this method cannot adapt, leading to gridlocks even at medium densities. The self-organizing method achieves optimality for low densities (no vehicle stops) and medium densities (all intersections are used at maximum capacity: there are always vehicles crossing all intersections. Topologically it is not possible to improve this). For other densities, the performance is close to the optimality curves (for details, see Gershenson and Rosenblueth, 2012).

More recently, we have found that self-organizing traffic lights would improve traffic more than if all vehicles were autonomous but with traditional traffic lights. Nevertheless, autonomous vehicles and self-organizing traffic lights are even better (Zapotecatl, 2019).

By distributing control locally, the requisite variety of the traffic light coordination can be tackled robustly as conditions change, while the formation of platoons self-organizes the traffic flows and assists the coordination of intersection controllers at the city scale. In this way, the traffic lights are mediators of vehicles, but the vehicles are also mediators of traffic lights. We have made simulations with up to ten thousand intersections achieving efficient or optimal coordination, so this solution is certainly scalable.

As there are so many variables involved in this system, simulations are necessary to explore and test potential solutions. It is natural to represent the topology of a city as a network, where nodes are intersections and links are streets connecting them. Vehicles and traffic lights can be usefully described as agents, since they act on their environment. It is worth noting that then traffic lights become part of the environment of vehicles, while vehicles are part of the environment of traffic lights.

\subsection{Public Transport Regulation}

In theory, passengers in public transport are served optimally when vehicle headway - the time between arrivals at a stationis equal. However, as we have shown, an equal headway configuration is unstable by nature (Gershenson and Pineda, 2009), since delays become amplified by positive feedbacks. Thus, many efforts have been made by transportation engineers to prevent the "equal headway instability," also known as the "bus bunching problem."

To keep equal headways, all vehicles-trains, trams, busesmust wait the same time at each station. This time can vary from station to station, but it must be fixed or some vehicles will go faster than others, leading to unequal headways and potentially to the collapse of the system. Since the precise number of passengers varies each time a vehicle reaches a station, and thus the required waiting time, then either vehicles will require a margin and be idle, or they will depart before servicing all passengers when these are more than expected.

We proposed a self-organizing algorithm inspired by ant colony communication (Gershenson, 2011a; Carreón et al., 2017), so this can be seen as an example of living technology. Some ant species communicate via their environment, a phenomenon known as stigmergy (Theraulaz and Bonabeau, 1999). When they find a food source, they return to their nest leaving a pheromone trail. This indicates the food location to other ants. When they find the food, they can reinforce the trail while returning to their nest. Since pheromones evaporate, once the food is finished, ants stop reinforcing the trail, and they start exploring again. In the case of our algorithm, vehicles can be seen as ants, and we wanted a pheromone-like environmental signal to be used to indicate when the last vehicle had passed. However, pheromones reduce their concentration, while we needed an increasing signal, so we defined "antipheromones" that are secreted by the environment, increase their concentration in time, and are erased by vehicles as they pass. 


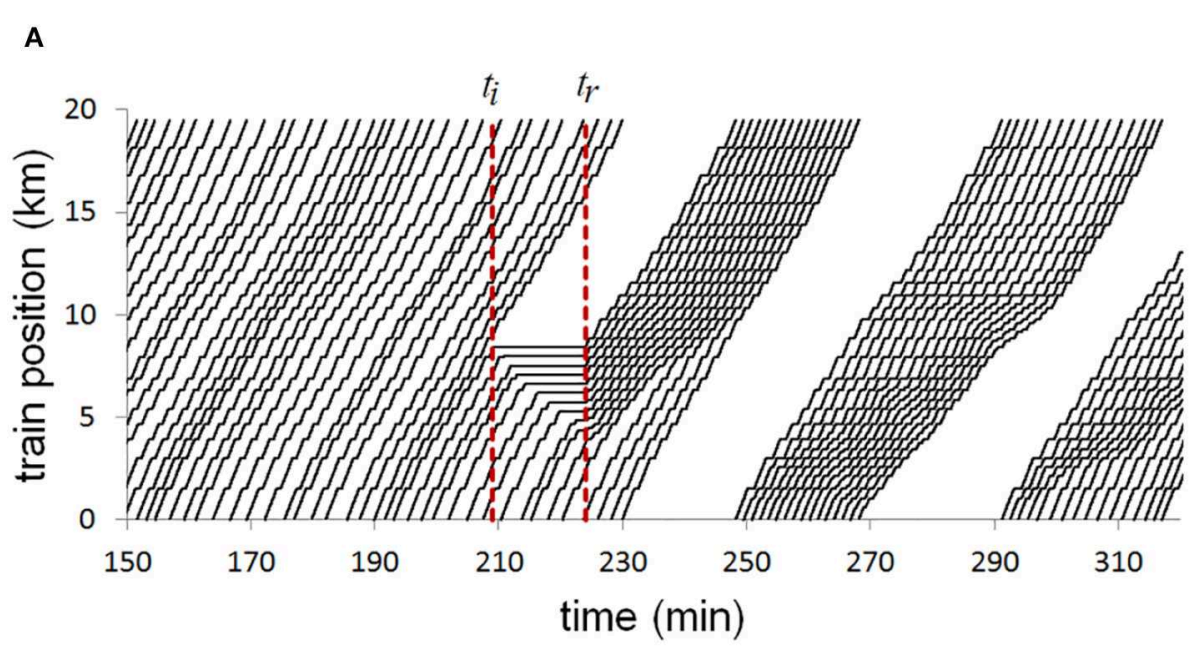

B

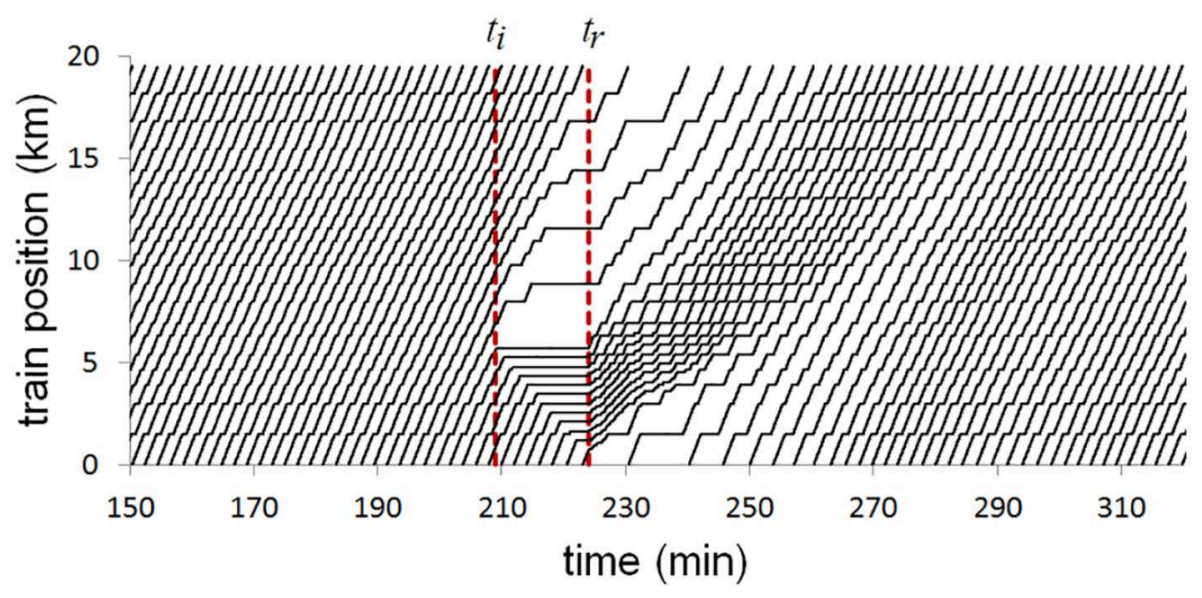

FIGURE 6 | Diagram showing positions of trains at different times. Trains move upwards in distance and rightwards in time. There is an interruption of service at $t_{i}$, and it is reestablished 15 min later at $t_{r}$. (A) Current control method. (B) Self-organizing method. Reproduced from Carreón et al. (2017) under the Creative Commons CCBY license https://doi.org/10.1371/journal.pone.0190100.g014.

In our algorithm, each vehicle "simply" tries to keep equal distance to the vehicles in front and behind (using antipheromones as mediators), but is flexible enough to serve passengers at stations and at the same time prevent idling. Equal headways are not maintained, but the system does not collapse. Rather, its performance is even better than the case with equal headways, i.e., it is supraoptimal. This is because of the slower-is-faster effect: It is true that passengers minimize their waiting time at stations with equal headways (as expected by theory). But their total travel time is not independent of the equal headways, so idling will increase their total travel time. With the self-organizing algorithm, passengers wait more at stations, but once they board a vehicle, they will reach their destination faster, as there is no idling. Again, adaptation takes place at the scales at which the system changes. We can say that this approach is antifragile, as supraoptimality is achieved precisely because of the "noise." (heterogeneity) of arriving passengers. If all stations had always the same demand (homogeneous), then the self-organizing algorithm would perform as good as the theoretical optimum, i.e., less than supraoptimal.

Figure 6 shows results from a simulation of Line 1 of the Mexico City Metro. On the top panel, the trajectories of trains using the current regulation method is depicted. There is a 15 min interruption of the service, and it can be seen that the system does not recover. In reality, the system does recover, but it requires human intervention and can take one, two, or more hours, depending on the passenger demand. On the bottom panel of Figure 6, the trajectories of a similar scenario are shown, but using our self-organizing method. It can be seen that even before the service is reestablished, the vehicles try to maintain equal headways with their neighbors, delaying vehicles ahead of the station where service was interrupted. Once service is reestablished, since the intervals between trains did not collapse, 
trains can quickly adapt and respond to the delayed service, recovering a desired configuration in less than half an hour.

\section{DISCUSSION}

We cannot reduce the complexity of several systems we have to deal with. Novel information produced by interactions leads to changes, making problems non-stationary. For example, in the case of traffic lights, one cannot try to optimize intersections in isolation and expect the system to be coordinated. Since the "output" of one intersection becomes the "input" of the next one downstream, this information should be constantly updated by sensors and taken into consideration by controllers.

Self-organization has been used in a broad variety of cyberphysical systems. It allows systems to adapt at the scales at which the problem they are solving changes in a robust fashion. In addition to the case studies mentioned in the previous section, dynamic road pricing in Singapore and variable parking cost in San Francisco are examples of self-organization being used to regulate urban mobility. We can see that the same principles apply in other cyber-physical and cyber-social systems, from telecommunications (Amoretti and Gershenson, 2016) to organizations (Gershenson, 2008).

As in the case of crowd control, there are many systems where we cannot change the components. Still, we can try to mediate interactions to control the function of the system. We will not change politicians. But perhaps we can regulate their interactions to improve politics. We cannot change teachers. But maybe novel mediators can improve education. Businesspeople will not change. But probably promoting certain interactions and restricting others can improve economies. It can take lots of energy to turn charcoal into diamond, but it can be done. They are made of the same atoms. "Only" their organization is different.

A relevant step toward adopting self-organizing controllers is to give up the desire to control completely our systems. This implies accepting that predictability is limited by complexity, and that adaptation should complement this inherent uncertainty, even if we do not know how systems will adapt. As complexity limits our predictability, systems require certain autonomy to make the "right decisions." Even if we use traditional approaches, we do not have full control of our systems, as they are constantly entering unexpected situations. We would like to be able to be sure that our systems will never fail, but they will. We can have formal proofs but these are also limited, since they assume idealized/closed/predefined situations. Self-organizing systems can do the same as traditional engineered systems and more, as they can deal with more realistic/open/variable situations. We just have to (systematically and cautiously) try and see, constantly adapting (Gershenson, 2007). Even if a solution already worked, it does not assure that it will continue working (as conditions change) or that it can be applied in the same way in a different context.

The best solution depends on the context/environment /problem. In some cases, centralized control will be good,
TABLE 1 | Different control approaches are more appropriate for different causalities, complexities, and diversities.

\begin{tabular}{llll}
\hline Control & Causality & Complexity & Diversity \\
\hline Centralized & Top-down & Low & Homogeneous or heterogeneous \\
Distributed & Bottom-up & Medium & Homogeneous \\
Self-organizing & Multiscale & High & Homogeneous or heterogeneous \\
\hline
\end{tabular}

TABLE 2 | Different control types are more related to certain concepts, approaches, and aspects.

\begin{tabular}{llll}
\hline Control & Concepts & Approaches & Aspects \\
\hline Active & Adaptation/antifragility & Agents & Functional \\
Passive & Robustness/heterogeneity & Networks & Structural \\
\hline
\end{tabular}

in others distributed is more appropriate, in yet others self-organizing. As shown in Table 1, centralized control is appropriate when causality should be top-down. Because of the law of requisite variety, systems with a high variety/complexity will require a controller with a high variety/complexity, so the centralized approach becomes less viable. Distributed control can deal with a greater complexity, but it is still limited, because the integration of the distributed solutions is not necessarily trivial. This limits distributed control to homogeneous systems: since information flow across the system is restricted, the local solutions assume that each local problem is similar. As illustrated in the traffic lights example, self-organizing control can deal with top-down and bottom-up causality (multiscale), as components can interact in a distributed fashion to change system properties (bottom-up), but then the system properties can mediate (top-down) to regulate the behavior of components. Self-organization can be scalable, adaptive, robust, and can deal with a high complexity and homogenous or heterogeneous problems. It is not that one approach is better than others, but they are more appropriate for different problems. Centralized control is easier to implement and understand, but is useful for low complexity/variety problems. Distributed control can deal with a greater complexity, but only for homogeneous, separable systems. Self-organizing systems might be more difficult to design and test, but they can handle greater complexity/variety/diversity.

How the control is organized is certainly relevant, but also whether the control is active or passive. As shown in Table 2, active control is more related with adaptation and antifragility, as these concepts imply constant change in the function of the controller. An agent-based approach is natural here, as it is straightforward to describe actions with agents, since these are entities that act on their environment. On the other hand, passive control is more related with robustness and heterogeneity, as these are intrinsic properties of systems and their structure (independently on whether there is change or not in the environment). A network description is useful in this case, as the relationships between elements can describe the organization of a system. Note that these are not exclusive, e.g., one can certainly use both active and passive controllers, or combine 
agents represented as networks, or study how structure and function affect each other. Also, the concepts and approaches not mentioned here apply to both control cases. Moreover, the relationship between structure and function is far from trivial and has been an open area of research (Heylighen, 1999), since structure defines function but also function can change structure. In many cases, we design structure for a desired function, but also we can design function for a desired structure (Dorigo et al., 2004; Werfel et al., 2014).

As the complexity of our cyber-physical systems increases, and also our understanding of it, we will see more self-organizing approaches. Perhaps names will differ, but the concepts presented here are required to control cyber-physical and cyber-social systems by guiding their self-organization.

\section{REFERENCES}

Albert, R., and Barabási, A.-L. (2002). Statistical mechanics of complex networks. Rev. Mod. Phys. 74, 47-97. doi: 10.1103/RevModPhys.74.47

Aldana, M. (2003). Boolean dynamics of networks with scale-free topology. Physica D 185, 45-66. doi: 10.1016/S0167-2789(03)00174-X

Amoretti, M. and Gershenson, C. (2016). Measuring the complexity of adaptive peer-to-peer systems. Peer Peer Netw. Appl. 9, 1031-1046.

Arthur, W. B. (1999). Complexity and the economy. Science 284, 107-109. doi: $10.1126 /$ science.284.5411.107

Ashby, W. R. (1947). Principles of the self-organizing dynamic system. J. Gen. Psychol. 37, 125-128. doi: 10.1080/00221309.1947.9918144

Ashby, W. R. (1956). An Introduction to Cybernetics. London: Chapman \& Hall. doi: 10.5962/bhl.title.5851

Ashby, W. R. (1962). "Principles of the self-organizing system," in Principles of Self-Organization, eds H. V. Foerster and G. W. Zopf Jr. (Oxford: Pergamon), 255-278.

Atlan, H. (1974). On a formal definition of organization. J. Theor. Biol. 45, 295-304. doi: 10.1016/0022-5193(74)90115-5

Axelrod, R. (1997). "Advancing the art of simulation in the social sciences," in Simulating Social Phenomena, Lecture Notes in Economics and Mathematical Systems, eds R. Conte, R. Hegselmann, and P. Terna (Berlin; Heidelberg: Springer), 21-40.

Ay, N., Der, R., and Prokopenko, M. (2012). Guided self-organization: perception-action loops of embodied systems. Theory Biosci. 131, 125-127. doi: 10.1007/s12064-011-0140-1

Balleza, E., Alvarez-Buylla, E. R., Chaos, A., Kauffman, S., Shmulevich, I., and Aldana, M. (2008). Critical dynamics in genetic regulatory networks: examples from four kingdoms. PLoS ONE 3:e2456. doi: 10.1371/journal.pone.0002456

Barabási, A.-L. (2016). Network Science. Cambridge: Cambridge University Press.

Barabási, A.-L., Albert, R., and Jeong, H. (2000). Scale-free characteristics of random networks: the topology of the world-wide web. Physica A 281, 69-77. doi: 10.1016/S0378-4371(00)00018-2

Bar-Yam, Y. (2004). Multiscale variety in complex systems. Complexity 9, 37-45. doi: $10.1002 /$ cplx.20014

Bedau, M. A., McCaskill, J. S., Packard, N. H., Parke, E. C., and Rasmussen, S. R. (2013). Introduction to recent developments in living technology. Artif. Life 19, 291-298. doi: 10.1162/ARTL_e_00121

Bedau, M. A., McCaskill, J. S., Packard, N. H., and Rasmussen, S. (2009). Living technology: exploiting life's principles in technology. Artif. Life 16, 89-97. doi: 10.1162/artl.2009.16.1.16103

Beer, R. D. (1990). Intelligence as Adaptive Behavior: An Experiment in Computational Neuroethology. San Diego, CA: Academic Press.

Benyus, J. M. (1997). Biomimicry: Innovation Inspired by Nature. New York, NY: William Morrow.

Bonabeau, E. (2002). Agent-based modeling: methods and techniques for simulating human systems. Proc. Natl. Acad. Sci. U.S.A. 99(Suppl. 3):72807287. doi: 10.1073/pnas.082080899

\section{AUTHOR CONTRIBUTIONS}

The author confirms being the sole contributor of this work and has approved it for publication.

\section{FUNDING}

This work was partially supported by UNAM's PAPIIT projects IN107919 and IV100120.

\section{ACKNOWLEDGMENTS}

I appreciate useful comments from János Kertész, special issue editors, and reviewers.

Brambilla, M., Ferrante, E., Birattari, M., and Dorigo, M. (2013). Swarm robotics: a review from the swarm engineering perspective. Swarm Intell. 7, 1-41. doi: 10.1007/s11721-012-0075-2

Caldarelli, G. (2007). Scale-Free Networks. Oxford: Oxford University Press.

Camazine, S., Deneubourg, J.-L., Franks, N. R., Sneyd, J., Theraulaz, G., and Bonabeau, E. (2003). Self-Organization in Biological Systems. Princeton, NJ: Princeton University Press.

Carreón, G., Gershenson, C., and Pineda, L. A. (2017). Improving public transportation systems with self-organization: a headway-based model and regulation of passenger alighting and boarding. PLoS ONE 12:e0190100. doi: 10.1371/journal.pone.0190100

Čejková, J., Banno, T., Hanczyc, M. M., and Štěpánek, F. (2017). Droplets as liquid robots. Artif. Life 23, 528-549. doi: 10.1162/ARTL_a_00243

Challet, D., Marsili, M., and Zhang, Y.-C. (2013). Minority Games: Interacting Agents in Financial Markets. Number 9780199686698 in OUP Catalogue. Oxford: Oxford University Press.

Cocho, G., Flores, J., Gershenson, C., Pineda, C., and Sánchez, S. (2015). Rank diversity of languages: generic behavior in computational linguistics. PLoS ONE 10:e0121898. doi: 10.1371/journal.pone.0121898

De Domenico, M., Camargo, C., Gershenson, C., Goldsmith, D., Jeschonnek, S., Kay, L., et al. (2019). Complexity Explained: A Grassroot Collaborative Initiative to Create a Set of Essential Concepts of Complex Systems. Available online at: https://complexityexplained.github.io

De Wolf, T., Samaey, G., and Holvoet, T. (2005). "Engineering self-organising emergent systems with simulation-based scientific analysis," in Proceedings of the International Workshop on Engineering Self-Organising Applications (Utrecht), 46-160.

Di Marzo Serugendo, G., Karageorgos, A., Rana, O. F., and Zambonelli, F., editors (2004). Engineering Self-Organising Systems, Nature-Inspired Approaches to Software Engineering, volume 2977 of Lecture Notes in Computer Science. Berlin; Heidelberg: Springer.

Dorigo, M., Trianni, V., Şahin, E., Groß, R., Labella, T. H., Baldassarre, G., et al. (2004). Evolving self-organizing behaviors for a swarm-bot. Auton. Robots 17, 223-245. doi: 10.1023/B:AURO.0000033973.24945.f3

Dressler, F. (2008). "Bio-inspired networking - self-organizing networked embedded systems," in Organic Computing, ed R. P. Würtz (Berlin; Heidelberg; New York, NY: Springer), 285-302.

Epstein, J. (2006). Generative Social Science: Studies in Agent-Based Computational Modeling. Princeton Studies in Complexity. Princeton, NJ: Princeton University Press.

Epstein, J. M., and Axtell, R. L. (1996). Growing Artificial Societies: Social Science from the Bottom Up. Washington, DC; Cambridge, MA: Brookings Institution Press; MIT Press.

Frei, R., and Di Marzo Serugendo, G. (2011). Advances in complexity engineering. Int. J. Bio-Inspired Comput. 3, 199-212. doi: 10.1504/IJBIC.2011.041144

Gershenson, C. (2001). Artificial societies of intelligent agents (B.E. thesis). Fundaciï $£_{j n}$ Arturo Rosenblueth, Mexico.

Gershenson, C. (2005). Self-organizing traffic lights. Comp. Syst. 16, 29-53. 
Gershenson, C. (2007). Design and Control of Self-organizing Systems. Mexico: CopIt Arxives. Available online at: http://tinyurl.com/DCSOS2007

Gershenson, C. (2008). Towards self-organizing bureaucracies. Int. J. Public Informat. Syst. 2008, 1-24.

Gershenson, C. (2011a). Self-organization leads to supraoptimal performance in public transportation systems. PLOS ONE 6:e21469. doi: 10.1371/journal.pone.0021469

Gershenson, C. (2011b). The sigma profile: a formal tool to study organization and its evolution at multiple scales. Complexity 16, 37-44. doi: 10.1002/cplx.20350

Gershenson, C. (2012). Guiding the self-organization of random Boolean networks. Theory Biosci. 131, 181-191. doi: 10.1007/s12064-011-0144-x

Gershenson, C. (2013a). "Facing complexity: prediction vs. adaptation," in Complexity Perspectives on Language, Communication and Society, eds A. Massip and A. Bastardas (Berlin; Heidelberg: Springer), 3-14.

Gershenson, C. (2013b). The implications of interactions for science and philosophy. Found. Sci. 18, 781-790. doi: 10.1007/s10699-012-9305-8

Gershenson, C. (2013c). Living in living cities. Artif. Life 19, 401-420. doi: 10.1162/ARTL_a_00112

Gershenson, C. (2015). Requisite variety, autopoiesis, and self-organization. Kybernetes 44, 866-873. doi: 10.1108/K-01-2015-0001

Gershenson, C., and Helbing, D. (2015). When slower is faster. Complexity 21, 9-15. doi: 10.1002/cplx.21736

Gershenson, C., and Heylighen, F. (2003). "When can we call a system selforganizing?," in Advances in Artificial Life, 7th European Conference, ECAL 2003 LNAI 2801, eds W. Banzhaf, T. Christaller, P. Dittrich, J. T. Kim, and J. Ziegler (Berlin: Springer), 606-614.

Gershenson, C., and Heylighen, F. (2005). "Chap. 3: How can we think the complex?," in Managing Organizational Complexity: Philosophy, Theory and Application, ed K. Richardson (Greenwich, CT: Information Age Publishing), 47-61.

Gershenson, C., and Pineda, L. A. (2009). Why does public transport not arrive on time? The pervasiveness of equal headway instability. PLoS ONE 4:e7292. doi: 10.1371/journal.pone.0007292

Gershenson, C., and Prokopenko, M. (2011). Complex networks. Artif. Life 17, 259-261. doi: 10.1162/artl_e_00037

Gershenson, C., and Rosenblueth, D. A. (2012). Self-organizing traffic lights at multiple-street intersections. Complexity 17, 23-39. doi: 10.1002/cplx.20392

Gershenson, C., Trianni, V., Werfel, J., and Sayama, H. (2018). "Self-organization and artificial life: a review," in The 2018 Conference on Artificial Life: A Hybrid of the European Conference on Artificial Life (ECAL) and the International Conference on the Synthesis and Simulation of Living Systems (ALIFE), eds T. Ikegami, N. Virgo, O. Witkowski, M. Oka, R. Suzuki, and H. Iizuka (Tokyo: MIT Press), 510-517.

Gibson, D. G., Glass, J. I., Lartigue, C., Noskov, V. N., Chuang, R.-Y., Algire, M. A., et al. (2010). Creation of a bacterial cell controlled by a chemically synthesized genome. Science 329, 52-56. doi: 10.1126/science.1190719

Goel, S., Bush, S. F., and Gershenson, C. (2017). Self-organization in traffic lights: evolution of signal control with advances in sensors and communications. arXiv [preprint] arXiv:1708.07188.

Haken, H. (1988). Information and Self-organization: A Macroscopic Approach to Complex Systems. Berlin: Springer-Verlag.

Helbing, D., Farkas, I., and Vicsek, T. (2000a). Simulating dynamical features of escape panic. Nature 407, 487-490. doi: 10.1038/35035023

Helbing, D., Farkas, I. J., and Vicsek, T. (2000b). Freezing by heating in a driven mesoscopic system. Phys. Rev. Lett. 84, 1240-1243. doi: 10.1103/PhysRevLett.84.1240

Helbing, D., Siegmeier, J., and Lämmer, S. (2007). Self-organized network flows. Netw. Heterogeneous Media 2, 193-210. doi: 10.3934/nhm.20 07.2.193

Heylighen, F. (1999). "The growth of structural and functional complexity during evolution," in The Evolution of Complexity Volume 8 of Einstein Meets Magritte, eds F. Heylighen, J. Bollen, and A. Riegler (Dordrecht: Kluwer), 17-44.

Heylighen, F. (2006). "Mediator evolution: a general scenario for the origin of dynamical hierarchies," in Worldviews, Science and Us, eds D. Aerts, B. D'Hooghe, and N. Note (Singapore: World Scientific), 45-48.

Heylighen, F., and Joslyn, C. (2001). "Cybernetics and second order cybernetics," in Encyclopedia of Physical Science and Technology, 3rd Edn., Vol. 4, ed R. A. Meyers (New York, NY: Academic Press), 155-170.
Heylighen, F., Joslyn, C., and Turchin, V. (1993). Principia Cybernetica Web. Available online at: http://pcp.vub.ac.be

Holland, J. H. (1975). Adaptation in Natural and Artificial Systems. Ann Arbor, MI: The University of Michigan Press.

Jen, E. (ed.). (2005). Robust Design: A Repertoire of Biological, Ecological, and Engineering Case Studies. Oxford: Santa Fe Institute Studies on the Sciences of Complexity, Oxford University Press.

Kauffman, S. A. (1993). The Origins of Order. Oxford: Oxford University Press.

Kriegman, S., Blackiston, D., Levin, M., and Bongard, J. (2020). A scalable pipeline for designing reconfigurable organisms. Proc. Natl. Acad. Sci. U.S.A. 117, 1853-1859. doi: 10.1073/pnas. 1910837117

Langton, C. G. (1990). Computation at the edge of chaos: phase transitions and emergent computation. Physica D 42, 12-37. doi: 10.1016/0167-2789(90)90064-V

Lazer, D., Pentland, A. S., Adamic, L., Aral, S., Barabasi, A. L., Brewer, D., et al. (2009). Life in the network: the coming age of computational social science. Science 323:721. doi: $10.1126 /$ science.1167742

Liu, J., and Tsui, K. C. (2006). Toward nature-inspired computing. Commun. ACM 49, 59-64. doi: 10.1145/1164394.1164395

Lloyd, S. (2001). Measures of Complexity: A Non-exhaustive List. Cambridge, MA: Department of Mechanical Engineering, Massachusetts Institute of Technology.

Maes, P. (1994). Modeling adaptive autonomous agents. Artif. Life 1, 135-162. doi: 10.1162/artl.1993.1.1_2.135

Mamei, M., Menezes, R., Tolksdorf, R., and Zambonelli, F. (2006). Case studies for self-organization in computer science. J. Syst. Architect. 52, 443-460. doi: 10.1016/j.sysarc.2006.02.002

Michod, R. E. (2003). "Cooperation and conflict mediation during the origin of multicellularity," in Genetic and Cultural Evolution of Cooperation, Ch. 16, ed P. Hammerstein (Cambridge, MA: MIT Press), 261-307.

Morales, J. A., Colman, E., Sánchez, S., Sánchez-Puig, F., Pineda, C., Iniguez, G., et al. (2018). Rank dynamics of word usage at multiple scales. Front. Phys. 6:45. doi: 10.3389/fphy.2018.00045

Müller-Schloer, C., Schmeck, H., and Ungerer, T. (eds.). (2011). Organic Computing - A Paradigm Shift for Complex Systems. Autonomic Systems. Basel: Springer.

Newman, M., Barabási, A.-L., and Watts, D. J., editors (2006). The Structure and Dynamics of Networks. Princeton Studies in Complexity. Princeton, NJ: Princeton University Press.

Newman, M. E. J. (2003). The structure and function of complex networks. SIAM Rev. 45, 167-256. doi: 10.1137/S003614450342480

Nicolis, G., and Prigogine, I. (1977). Self-Organization in Non-Equilibrium Systems: From Dissipative Structures to Order Through Fluctuations. Chichester: Wiley.

Pagels, H. R. (1989). The Dreams of Reason: The Computer and the Rise of the Sciences of Complexity. New York, NY: Bantam Books.

Pineda, O. K., Kim, H., and Gershenson, C. (2019). A novel antifragility measure based on satisfaction and its application to random and biological Boolean networks. Complexity 2019:10. doi: 10.1155/2019/37 28621

Polani, D., Prokopenko, M., and Yaeger, L. S. (2013). Information and self-organization of behavior. Adv. Comp. Syst. 16:1303001. doi: $10.1142 / \mathrm{S} 021952591303001 \mathrm{X}$

Prokopenko, M. (2009). Guided self-organization. HFSP J. 3, 287-289. doi: $10.2976 / 1.3233933$

Prokopenko, M. (ed.). (2014). Guided Self-Organization: Inception, volume 9 of Emergence, Complexity and Computation. Berlin; Heidelberg: Springer.

Prokopenko, M., Boschetti, F., and Ryan, A. (2009). An information-theoretic primer on complexity, self-organisation and emergence. Complexity 15, 11-28. doi: $10.1002 /$ cplx.20249

Rahwan, I., Cebrian, M., Obradovich, N., Bongard, J., Bonnefon, J.-F., Breazeal, C., et al. (2019). Machine behaviour. Nature 568, 477-486. doi: 10.1038/s41586-019-1138-y

Rasmussen, S., Bedau, M. A., Chen, L., Deamer, D., Krakauer, D. C., Packard, N. H., et al. (eds.). (2008). Protocells: Bridging Nonliving and Living Matter. Cambridge, MA: MIT Press.

Rohden, M., Sorge, A., Timme, M., and Witthaut, D. (2012). Self-organized synchronization in decentralized power grids. Phys. Rev. Lett. 109:064101. doi: 10.1103/PhysRevLett.109.064101 
Rubenstein, M., Cornejo, A., and Nagpal, R. (2014). Programmable self-assembly in a thousand-robot swarm. Science 345, 795-799. doi: 10.1126/science. 1254295

Schweitzer, F. (2003). Brownian Agents and Active Particles. Collective Dynamics in the Natural and Social Sciences. Springer Series in Synergetics. Berlin: Springer.

Simon, H. A. (1996). The Sciences of the Artificial, 3rd Edn. Cambridge, MA: MIT Press.

Steels, L. (1993). "Building agents out of autonomous behavior systems," in The Artificial Life Route to Artificial Intelligence: Building Embodied Situated Agents, eds L. Steels and R. A. Brooks (New Haven, CT: Lawrence Erlbaum), 102-137.

Steels, L., and Brooks, R. (1995). The Artificial Life Route to Artificial Intelligence: Building Embodied, Situated Agents. New York, NY: Lawrence Erlbaum Associates.

Taleb, N. N. (2012). Antifragile: Things That Gain From Disorder. London: Random House.

Theraulaz, G., and Bonabeau, E. (1999). A brief history of stimergy. Artif. Life 5, 97-116. doi: 10.1162/106454699568700

Vásárhelyi, G., Virágh, C., Somorjai, G., Nepusz, T., Eiben, A. E., and Vicsek, T. (2018). Optimized flocking of autonomous drones in confined environments. Sci. Robot. 3:20. doi: 10.1126/scirobotics.aat3536

Vicsek, T., and Zafeiris, A. (2012). Collective motion. Phys. Rep. 517, 71-140. doi: 10.1016/j.physrep.2012.03.004

von Bertalanffy, L. (1968). General System Theory: Foundations, Development, Applications. New York, NY: George Braziller.

von Foerster, H. (1960). "On self-organizing systems and their environments," in Self-Organizing Systems, eds M. C. Yovitts and S. Cameron (New York, NY: Pergamon), 31-50.

Wagner, A. (2005). Robustness and Evolvability in Living Systems. Princeton, NJ: Princeton University Press.

Werfel, J., Petersen, K., and Nagpal, R. (2014). Designing collective behavior in a termite-inspired robot construction team. Science 343, 754-758. doi: $10.1126 /$ science. 1245842

Wilensky, U., and Rand, W. (2015). An Introduction to Agent-Based Modeling: Modeling Natural, Social, and Engineered Complex Systems with NetLogo. Cambridge, MA: MIT Press.
Wolfram, S. (2002). A New Kind of Science. Champaign, IL: Wolfram Media.

Wooldridge, M., and Jennings, N. R. (1995). Intelligent agents: theory and practice. Knowl. Eng. Rev. 10, 115-152. doi: 10.1017/S02698889000 08122

Wuensche, A., and Lesser, M. (1992). The Global Dynamics of Cellular Automata; An Atlas of Basin of Attraction Fields of One-Dimensional Cellular Automata. Reading, MA: Santa Fe Institute Studies in the Sciences of Complexity. Addison-Wesley.

Zambonelli, F., and Rana, O. F. (2005). Self-organization in distributed systems engineering: introduction to the special issue. IEEE Trans. Syst. Man Cybernet. Part A 35, 313-315. doi: 10.1109/TSMCA.2006.846372

Zapotecatl, J. L. (2019). Algoritmos de control de semáforos auto-organizados para incrementar el flujo de tránsito vehicular basados en autómatas celulares (Ph.D. thesis). Universidad Nacional Autónoma de México, Mexico.

Zapotecatl, J. L., Rosenblueth, D. A., and Gershenson, C. (2017). Deliberative self-organizing traffic lights with elementary cellular automata. Complexity 2017:7691370. doi: 10.1155/2017/7691370

Zubillaga, D., Cruz, G., Aguilar, L. D., Zapotécatl, J., Fernández, N., Aguilar, J., et al. (2014). Measuring the complexity of self-organizing traffic lights. Entropy 16, 2384-2407. doi: 10.3390/e16052384

Conflict of Interest: The author declares that the research was conducted in the absence of any commercial or financial relationships that could be construed as a potential conflict of interest.

The reviewer PZ declared a past co-authorship with one of the authors CG to the handling editor.

Copyright $\odot 2020$ Gershenson. This is an open-access article distributed under the terms of the Creative Commons Attribution License (CC BY). The use, distribution or reproduction in other forums is permitted, provided the original author(s) and the copyright owner(s) are credited and that the original publication in this journal is cited, in accordance with accepted academic practice. No use, distribution or reproduction is permitted which does not comply with these terms. 